\title{
Clinically relevant circulating microRNA profiling studies in pancreatic cancer using meta-analysis
}

\author{
Zenglin Pei ${ }^{1}$, Song-Mei Liu ${ }^{2}$, Jing-Tao Huang ${ }^{2}$, Xuan Zhang ${ }^{1}$, Dong Yan ${ }^{3}$, Qianlin \\ $\mathrm{Xia}^{1}$, Chunxia $\mathrm{Ji}^{1}$, Weiping Chen ${ }^{4}$, Xiaoyan Zhang ${ }^{1}$, Jianqing $\mathrm{Xu}^{1}$, Jin Wang ${ }^{1}$ \\ ${ }^{1}$ Scientific Research Center, Shanghai Public Health Clinical Center, Fudan University, Jinshan District, Shanghai 201508, P.R. China \\ ${ }^{2}$ Center for Gene Diagnosis, Zhongnan Hospital of Wuhan University, Wuhan, Hubei 430071, P.R. China \\ ${ }^{3}$ Department of Medical Oncology, Beijing Chaoyang Hospital affiliated to Capital Medical University, Beijing, China \\ ${ }^{4}$ Genomics Core, National Institute of Diabetes and Digestive and Kidney Diseases, National Institutes of Health, Bethesda, \\ MD 20892, USA
}

Correspondence to: Jin Wang, email: wangjin@shaphc.org

Keywords: pancreatic cancer, meta-analysis, diagnostics, multiple miRNA, SROC

Received: October 24, $2016 \quad$ Accepted: January 25, $2017 \quad$ Published: February 07,2017

\section{ABSTRACT}

Background: Pancreatic cancer ( $\mathrm{PaCa}$ ) is the most lethal gastrointestinal (GI) tumor. Although many studies on differentially expressed miRNAs as candidate biomarkers of pancreatic cancer have been published, reliability of these findings generated from investigations performed in single laboratory settings remain unclear.

Results: There were 29 articles with a total of 2,225 patients and 1,618 controls included in this meta-analysis. The pooled sensitivity was $82 \%$ (95\% CI, $79-85 \%)$; the specificity was $85 \%(95 \%$ CI, 79-89\%); and area under the curve (AUC) was $0.89(95 \% \mathrm{CI}, 0.86-0.92)$. Subgroup analyses indicated that there were significant divergences between Caucasian and Asian subgroups for circulating miRNA analysis.

Materials And Methods: To comprehensively investigate the potential utility of miRNAs as biomarkers of the disease, we searched publications diagnosing $\mathrm{PaCa}$ using miRNAs from PubMed, Medline, Embase, Google Scholar and Chinese National Knowledge Infrastructure (CNKI) databases. The sensitivity (SEN), specificity (SPE), and summary receiver operating characteristic (SROC) curve were used to examine the overall test performance, and heterogeneity was analyzed with the $I^{2}$ test.

Conclusions: Our analysis demonstrated that multiple miRNAs (SEN: 85\%; SPE: 89\%; AUC: 0.93) were more accurate for diagnosing PaCa than a single miRNA (SEN: 78\%; SPE: 79\%; AUC: 0.84), and future studies are still needed to confirm the diagnostic value of these pooled miRNAs for PaCa.

\section{INTRODUCTION}

Pancreatic cancer $(\mathrm{PaCa})$ is one of the most lethal and aggressive cancers, with most patients dying within one year after diagnosis and a less than 6\% 5-year survival rate [1]. However, the 5-year survival rate for pancreatic cancer patients increases significantly with curative resection of early-stage disease [2]. K-ras, p53, serum CA19-9 and CEA have been the most widely used biomarkers for PaCa diagnosis [3, 4], but these biomarkers often lead to incorrect diagnosis for $\mathrm{PaCa}$ and other noncancer pancreatic diseases (e.g., chronic pancreatitis) because of their unreliable sensitivity (SEN) and improper specificity (SPE) $[4,5]$, so that diagnosis of $\mathrm{PaCa}$ remains a major clinical challenge. There is therefore an urgent need to identify sensitive and specific biomarkers for early detection of pancreatic cancer. Thus, finding valid, reliable biomarkers for early detection and developing an objective molecular test for $\mathrm{PaCa}$ diagnosis will have clear clinical significance.

miRNA is a class of functional double stranded 18-24 nucleotide non-coding RNA molecules that decrease gene expression through translational inhibition or degeneration of target mRNA [6]. Tumor-associated miRNAs activate critical cancer relevant pathways and play key roles in the oncogenic process and are confirmed to be involved in the genetic networks regulating functional pathways in pancreatic cancer, which can 
be candidate biomarkers of $\mathrm{PaCa}[7,8]$. Although their diagnostic accuracy has been evaluated and several studies have obtained promising results, the possible application of miRNAs for diagnosing $\mathrm{PaCa}$ remains controversial due to wide-ranging values of SEN and SPE in these studies, which may lead to different results dependent on subjects' ethnicities, sources of controls, types of miRNAs, and specimen. Relatively low diagnostic accuracy was found in studies applying single-miRNA profiling for the diagnosis of PaCa. For example, Zhao et al. investigated the value of miR-192 for the diagnosis of $\mathrm{PaCa}$ with an SEN of 76.0\% and an SPE of 55.0\% in an Asian population [9]. Carlsen et al. investigated the value of miR-375 for the diagnosis of pancreatic ductal adenocarcinoma (PDAC) with SEN of $77.0 \%$ and SPE of $66.0 \%$ [10], which revealed that in the plasma-miRNA population, miR-375 was increased in PDAC cases compared with patients with other pancreatic or gastrointestinal diseases. The diagnostic accuracy of miR-21 for $\mathrm{PaCa}$ was confirmed in another Asian population, with results exhibiting an SEN of $77.8 \%$ and an SPE of $66.7 \%$, respectively [11]. Habbe et al. showed an SEN of $81.0 \%$ and an SPE of $98.0 \%$ in a Caucasian population for the diagnostic accuracy of miR-21 [12]. Moreover, Cote et al. investigated a set of miRNAs (miR-10b, miR-155, miR-106b, miR-30c, and miR-212) as biomarkers for the early diagnosis of $\mathrm{PaCa}$ with 95.0\% SEN and 100.0\% SPE [13]. We were motivated by these discordant results, which were generated from investigations performed in single laboratory settings with minimal evidence of reproducibility and independent validation in other laboratories [14], to conduct a metaanalysis to develop the diagnostic accuracy of miRNA assays for $\mathrm{PaCa}$ diagnosis.

\section{RESULTS}

\section{Systematic review and quality assessment of diagnostic studies of pancreatic cancer for meta- analysis}

Based on our primary literature research from PubMed, Medline, Embase, Google Scholar and Chinese National Knowledge Infrastructure (CNKI) databases, there are a total of 354 eligible relevant studies diagnosing $\mathrm{PaCa}$ using miRNAs in patients and an additional 23 eligible studies found by scanning the reference lists in our initial study, of which 25 studies were removed as duplicate records (Figure 1A). After screening the titles, abstracts and keywords, we further excluded 294 studies as reviews $(n=29)$, for the study not including miRNAs $(n=194)$, for the study not including PaCa diagnosis $(n=82)$ and lacking complete data $(n=18)$. Ultimately, there are 29 articles with 36 studies [2, 7-13, 15-35] published between 2009 and 2016 examining the efficacy of miRNAs for diagnosing PaCa compared with healthy controls and included a total of 3843 participants (2225 patients with $\mathrm{PaCa}$ and 1618 controls) from the United States, Japan, Germany, France, Denmark, and China, shown in Table 1. Habbe et al.'s article [12], Que et al.'s article [23], Cote et al.'s article [13], Xie et al.'s article [33], Humeau et al.'s article [7], Cao et al.'s article [2] and $\mathrm{Xu}$ et al.'s article [35] included 2 studies, and the remaining 21 articles included 1 study each [8-11, 15-22, 24-32, 34]. Next, we found that 21 studies were performed in Asian populations and the other 15 studies were performed in Caucasian populations. A total of 25 studies detected miRNA in blood (such as whole blood [24, 26, 28], serum [9, 20, 22, 23, 27, 29, 32, 34] and plasma samples $[2,8,10,11,13,15,16,18,19,25,35])$, and 11 studies detected miRNA in non-blood samples (including bile [13], cyst fluid [17], pancreatic juice [12, 30], salivary $[7,33]$, and stool $[21,31])$. We evaluated 19 studies for assessing the diagnostic efficacy of multiple miRNAs $[2,13,15,17-22,26-32,34]$ and single miRNAs using meta-analysis for discriminating patients with $\mathrm{PaCa}$ from healthy controls in 17 studies [7-12, 16, 23-25, 33, 35] in these 29 articles. The quantitative real-time polymerase chain reaction (qRT-PCR) or immunohistochemistry (IHC) assay were used in these studies for measuring the expression levels of these miRNAs, and the reference miRNAs (Table 1) was used as the endogenous control for normalization, such as RNU6B, RNU44, RNU48, miR-16, miR-24, miR-39, miR-54, miR-238, miR-425-5p, and miR-3196. Moreover, we found that RNU6B, miR16 , and miR-39 were often used as reference miRNAs for miRNAs based studies in pancreatic cancer. The qualities of the selected studies all turned out to be high according to QUADAS-2 guidelines (Figure 1B).

\section{Sensitivity and specificity of circulating miRNAs for the diagnosis of pancreatic cancer}

The overall pooled SEN and SPE for 36 studies were $82 \%(95 \%$ CI, 79-85\%) and 85\% (95\% CI, 79-89\%), respectively, for distinguishing patients with $\mathrm{PaCa}$ from healthy controls (Figure 2A and 2B). Meanwhile, significant heterogeneity was observed in SEN and SPE since $I^{2}$ for SEN was $76.34 \%\left(95 \%\right.$ CI, 68.82-83.85), and $I^{2}$ for SPE was $82.75 \%(95 \% \mathrm{CI}, 77.73-87.77)$. We therefore used the random-effect model for analysis. The PLR and NLR were 5.44 (95\% CI, 3.85-7.68) and 0.21 (95\% CI, $0.17-0.26$ ), respectively (Supplementary Figure $1 \mathrm{~A}$ and 1B), and the DOR was 29.95 (95\% CI, 15.69-42.94) (Supplementary Figure 2). Figure 3A showed the summary receiver operating characteristic (SROC) curve, and the AUC of these 36 studies was 0.89 (95\% CI, 0.86-0.92). Fagan's nomogram for likelihood ratios is shown in Supplementary Figure 3.

Next, pooled SEN, SPE, and AUC for 25 studies in which miRNA was measured in blood were $81 \%(95 \%$ CI, 77-85\%), 84\% (95\% CI, 79-88\%), and 0.89 (95\% CI, 0.86-0.92), respectively; SEN, SPE, and AUC in 
Table 1: The main features of included studies in meta-analysis

\begin{tabular}{|c|c|c|c|c|c|c|c|c|c|c|c|}
\hline \multirow{2}{*}{ Reference } & \multirow{2}{*}{ Ethnicity } & \multirow{2}{*}{ Specimen } & \multicolumn{2}{|c|}{ PCa } & \multicolumn{2}{|c|}{ Control } & \multirow{2}{*}{ Patient spectrum } & \multirow{2}{*}{ miRNA } & \multirow{2}{*}{$\begin{array}{c}\text { Reference } \\
\text { miRNA }\end{array}$} & \multirow{2}{*}{$\begin{array}{l}\text { Sensitivity } \\
(\%)\end{array}$} & \multirow{2}{*}{$\begin{array}{l}\text { Specificity } \\
(\%)\end{array}$} \\
\hline & & & Number & Age & Number & Age & & & & & \\
\hline \multirow{2}{*}{ Habbe et al. 2009} & \multirow{2}{*}{ Caucasian } & \multirow{2}{*}{ Pancreatic juice } & \multirow{2}{*}{64} & \multirow{2}{*}{ n.a. } & \multirow{2}{*}{54} & \multirow{2}{*}{ n.a. } & \multirow{2}{*}{ Pancreatic cancer } & miR-155 & \multirow{2}{*}{ RNU6B } & 83 & 93 \\
\hline & & & & & & & & miR-21 & & 81 & 98 \\
\hline Wang et al. 2009 & Caucasian & Plasma & 28 & n.a. & 19 & n.a. & PDAC & $\begin{array}{l}\text { miR-21, miR-210, miR-155, and } \\
\text { miR-196a }\end{array}$ & miR-16 & 64 & 89 \\
\hline Liu et al. 2011 & Asian & Plasma & 45 & n.a. & 30 & n.a. & PDAC & miR-21 & miR-39 & 77.8 & 66.7 \\
\hline Morimura et al. 2011 & Asian & Plasma & 36 & 68 & 30 & 58 & Pancreatic cancer & miR-18a & RNU6B & 90 & 75 \\
\hline Ryu et al. 2011 & Caucasian & $\begin{array}{c}\text { Pancreatic cyst } \\
\text { fluid } \\
\end{array}$ & 24 & 57 & 16 & 58 & Pancreatic cancer & miR-21, miR-221, miR-17-3p & RNU6B & 80 & 76 \\
\hline Bauer et al. 2012 & Caucasian & Plasma & 45 & n.a. & 33 & n.a. & PDAC & a set of 100 miRNAs & RNU6B & 100 & 93.7 \\
\hline Liu et al. 2012 & Asian & Plasma & 138 & 62 & 175 & 64 & Pancreatic cancer & $\begin{array}{l}\text { miR-16, miR-21, miR-155, miR- } \\
\text { 181a, miR-181b, miR-196a and } \\
\text { miR-210 }\end{array}$ & miR-39 & 64.5 & 78.9 \\
\hline Liu et al. 2012 & Asian & Serum & 95 & 63 & 81 & 58 & Pancreatic cancer & $\begin{array}{l}\text { miR-20a, miR-21, miR-24, miR- } \\
25, \text { miR-99a, miR-185 and miR- } \\
191\end{array}$ & - & 89 & 100 \\
\hline Ren et al. 2012 & Asian & Stool & 29 & 63 & 13 & 58 & Pancreatic cancer & miR-210, miR-196a, miR-181b & miR-16 & 84.6 & 69.2 \\
\hline $\begin{array}{l}\text { Kawaguchi et al } \\
2013\end{array}$ & Asian & Plasma & 47 & n.a. & 30 & n.a. & Pancreatic cancer & miR-221 & RNU6B & 74 & 78 \\
\hline Li et al. 2013 & Caucasian & Serum & 41 & 65 & 19 & 44 & PDAC & miR-1290 and miR-146a & miR-16 & 88 & 84 \\
\hline Wang et al. 2013 & Asian & Blood & 129 & 63 & 163 & 44 & \begin{tabular}{|l|} 
Pancreatic cancer \\
\end{tabular} & $\operatorname{miR}-27 a-3 p$ & RNU6B & 82.2 & 76.7 \\
\hline \multirow{2}{*}{ Que et al. 2013} & \multirow{2}{*}{ Asian } & \multirow{2}{*}{ Serum } & \multirow{2}{*}{22} & \multirow{2}{*}{65} & 27 & 58 & PDAC & miR-21 & RNU6B & 95.5 & 81.5 \\
\hline & & & & & & 50 & PDAC & miR-17-5p & RNUOD & 72.7 & 92.6 \\
\hline Zhao et al. 2013 & Asian & Serum & 70 & 60 & 40 & 60 & PDAC & miR-192 & RNU6B & 76 & 55 \\
\hline Carlsen et al. 2013 & Caucasian & Plasma & 47 & 65.5 & 45 & 59.5 & PDAC & miR-375 & $\begin{array}{l}\text { RNU6B, miR- } \\
238, \mathrm{miR}-54\end{array}$ & 77 & 66 \\
\hline Ganepola et al. 2014 & Caucasian & Blood & 11 & 68 & 11 & 46 & Pancreatic cancer & $\begin{array}{l}\text { miR-642b, miR-885-5p, miR- } \\
22-3 p\end{array}$ & miR-3196 & 91 & 91 \\
\hline Schultz et al. 2014 & Caucasian & Blood & 180 & 65 & 199 & 53 & Pancreatic cancer & $\begin{array}{l}\text { miR-26b, miR-34a, miR-122, } \\
\text { miR-126, miR-145, miR-150, } \\
\text { miR-223, miR-505, miR-636 and } \\
\text { miR-885.5p }\end{array}$ & $\begin{array}{l}\text { RNU44, } \\
\text { RNU48 }\end{array}$ & 85 & 85 \\
\hline Zhang et al. 2014 & Asian & Serum & 70 & n.a. & 40 & n.a. & PDAC & miR-192 and miR-194 & RNU6B & 84 & 75 \\
\hline Slater et al. 2014 & Caucasian & Serum & 9 & n.a. & 10 & n.a. & PDAC & miR-196a, miR-196b & miR-24 & 89 & 90 \\
\hline Wang et al. 2014 & Caucasian & Pancreatic juice & 50 & n.a. & 38 & n.a. & PDAC & $\begin{array}{c}\text { miR-205, miR-210, miR-492, } \\
\text { miR-1427 }\end{array}$ & RNU6B & 87 & 88 \\
\hline Cote et al 2014 & Caucasian & Plasma & 40 & 67 & 25 & 66 & PDAC & $\begin{array}{l}\text { miR-10b, miR-155, miR-106b, } \\
\text { miR-30c and miR-212 }\end{array}$ & & 95 & 100 \\
\hline Cote et al. 2014 & Caucasian & Bile & 40 & 67 & 25 & 66 & PDAC & $\begin{array}{l}\text { miR-10b, miR-155, miR-106b, } \\
\text { miR-30c and miR-212 }\end{array}$ & $\mathrm{miR}-42 \mathrm{~s}-5 \mathrm{p}$ & 96 & 100 \\
\hline Chen et al. 2014 & Asian & Plasma & 109 & n.a. & 50 & n.a. & Pancreatic cancer & miR-182 & RNU6B & 64.1 & 82.6 \\
\hline Lin et al. 2014 & Asian & Serum & 49 & 62 & 27 & 61 & \begin{tabular}{|l|} 
Pancreatic cancer \\
\end{tabular} & miR-492, miR-663a & miR-39 & 75.5 & 70 \\
\hline Yang et al. 2014 & Asian & Stool & 30 & n.a. & 25 & n.a. & PDAC & miR-21, miR-155, miR-216 & RNU6B & 83.33 & 83.33 \\
\hline Xie etal 2015 & Asian & Salivary & 40 & $n$ & 40 & $n a$ & Pancreatic cancer & $\operatorname{miR}-3679-5 p$ & RNU6B & 82.5 & 45 \\
\hline X1e et al. 2015 & Asian & salivary & 40 & n.a. & 40 & n.a. & Pancreatic cancer & miR-940 & RNUEB & 90 & 40 \\
\hline Humeau et al 2015 & Caucasian & Salivary & 7 & 67 & 4 & 70 & PDAC & miR-21 & - & 71.4 & 100 \\
\hline & & & & & & & & $\operatorname{miR}-23$ & - & 85.7 & 100 \\
\hline Kojima et al 2015 & Asian & Serum & 100 & n.a. & 21 & n.a. & Pancreatic cancer & $\begin{array}{c}\text { miR-6075, miR-4294, miR-6880- } \\
\text { 5p, miR-6799-5p, miR-125a-3p, } \\
\text { miR-4530, miR-6836-3p and } \\
\text { miR-4476 }\end{array}$ & - & 80.3 & 97.6 \\
\hline & & Plasma & 156 & n.a. & 57 & n.a. & Pancreatic cancer & $\begin{array}{c}\text { miR-486-5p, miR-126-3p, miR- } \\
106 b-3 p\end{array}$ & & 82.7 & 84.4 \\
\hline Cao et al. 2016 & Asian & Plasma & 29 & n.a. & 16 & n.a. & Pancreatic cancer & $\begin{array}{l}\text { miR-486-5p, miR-126-3p, miR- } \\
\text { 106b-3p, miR-938, miR-26b-3p, } \\
\text { and miR-1285 }\end{array}$ & RNU6B & 83.9 & 80.8 \\
\hline Xu et al 2016 & Asian & Plasma & 156 & $n \cdot a$ & 65 & $\mathrm{n} \cdot \mathrm{a}$ & Pancreatic cancer & miR-938 & RNU6B & 61.5 & 73.8 \\
\hline Auet al 2010 & Astan & Prasma & 100 & 11.a. & 03 & 11.a. & Pancreatic cancer & miR-486-5p & & 75 & 87.7 \\
\hline
\end{tabular}

11 studies which measured miRNA in other body fluids (such as stool, bile, pancreatic cyst fluid, pancreatic juice, and salivary) were $85 \%$ (95\% CI, $81-88 \%), 87 \%(95 \%$ $\mathrm{CI}, 70-95 \%)$, and 0.85 (95\% CI, 0.82-0.88) respectively (Table 2).

\section{Diagnostic efficacy of multiple miRNAs and a single miRNA in PaCa}

Evaluating the diagnostic accuracy of multiple miRNAs in the 19 studies, we found that the SEN was $85 \%$ (95\% CI, 80-89\%) (Figure 4C), the SPE was $89 \%$ (95\% CI, 83-92\%) (Figure 4D), and the AUC was 0.93 (95\% CI, 0.90-0.95) (Supplementary Figure 4B); for the diagnostic accuracy of a single miRNA in the 17 studies, we found that the SEN was $78 \%(95 \%$ CI, $74-82 \%)$ (Figure 4A), the SPE was $79 \%(95 \%$ CI, 69-86\%) (Figure 4B), and the AUC was 0.84 (95\% CI, 0.80-0.87) (Supplementary Figure 4A), which showed significant divergences between multiple miRNAs and single miRNA, indicating that multiple miRNA profiling is more accurate in diagnosing $\mathrm{PaCa}$.

\section{Ethnic subgroup analysis in pancreatic cancer based on circulating miRNA profiles}

Subgroup analyses were also conducted and the pooled results are shown in Table 2. Different ethnic 
subgroup analysis revealed that in the 20 studies the accuracy of miRNAs to differentiate $\mathrm{PaCa}$ from controls, the SEN was $80 \%$ (95\% CI, 76-83\%), the SPE was $83 \%$ (95\% CI, 75-89\%), and the AUC was 0.86 (95\% CI, 0.83-0.89). However, in the 16 studies for patients with PDAC, the SEN was $87 \%$ (95\% CI, 81-91\%), the SPE was $86 \%$ (95\% CI, 78-92\%), and the AUC was $0.93(95 \%$ CI, 0.90-0.95), indicating a higher accuracy compared with applications on $\mathrm{PaCa}$ patients. Subgroup analysis in the 21 studies conducted in Asian populations, the SEN and SPE were 79\% (95\% CI, 75-83\%) and 79\% (95\% CI, 71-85\%), the AUC was 0.85 (95\% CI, 0.82-0.88); for the 15 studies performed in Caucasian populations, these values were $87 \%$ (95\% CI, 81-91\%), 91\% (95\% CI, 85-95\%) and 0.94 (95\% CI, 0.92-0.96), which showed that there were significant divergences between Caucasian and Asian subgroups for circulating miRNA analysis.

\section{DISCUSSION}

Due to increasing incidence, mortality and low survival rates of $\mathrm{PaCa}$, screening is an urgent clinical challenge. However, accurate, affordable and repeatable detection methods are lacking. Even though miRNAs may have high diagnostic value [36], the clinical utility of miRNA for diagnosing pancreatic cancer remains disputable.
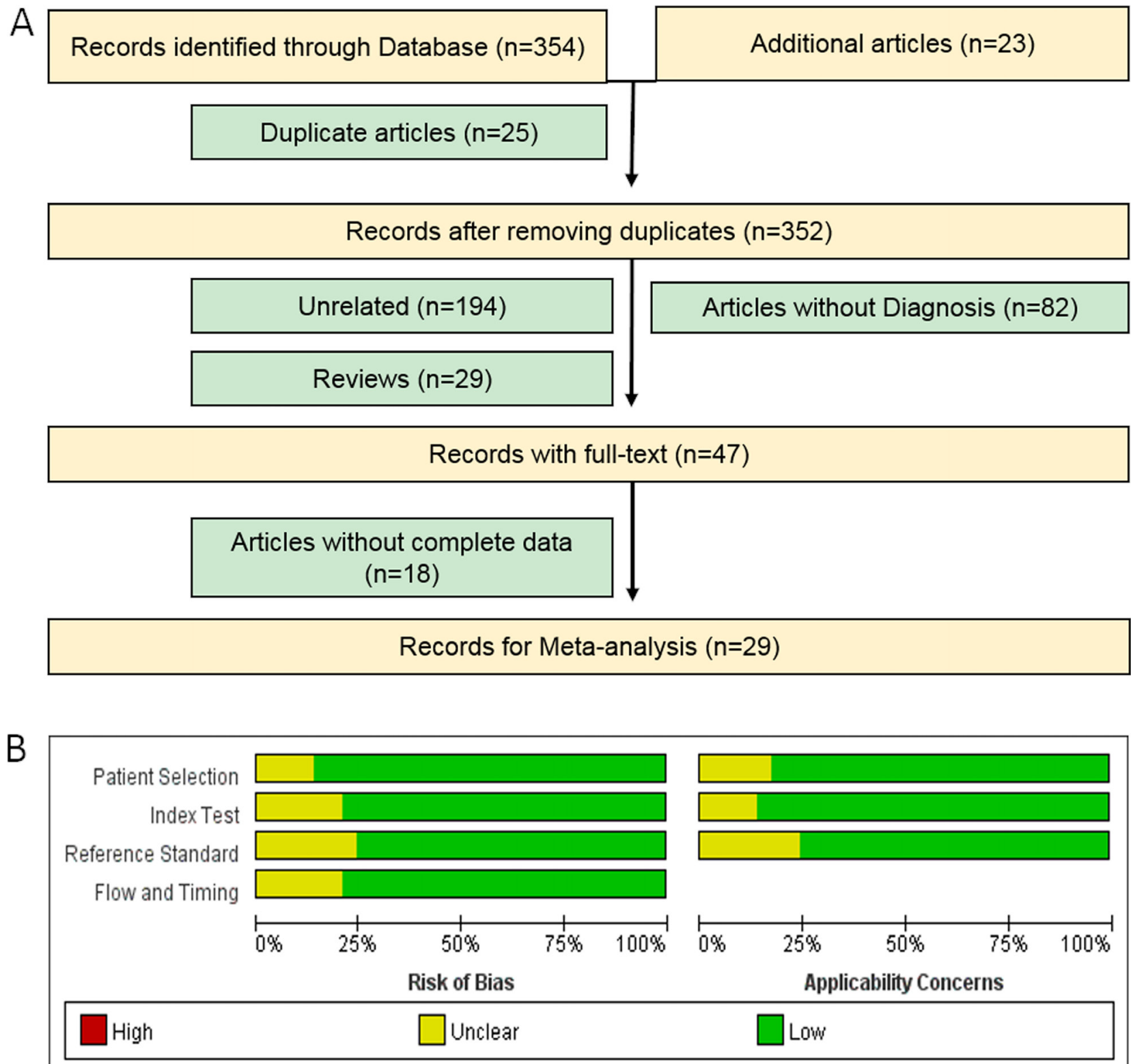

Figure 1: Flow chart of the meta-analysis of miRNA in $\mathrm{PaCa}(\mathbf{A})$ and quality of included studies according to QUADAS-2 guidelines: proportion of studies with risk of bias; proportion of studies with regarding applicability (B). 
Table 2: Summary estimates of subgroup analysis for miRNA in the diagnosis of pancreatic cancer

\begin{tabular}{|c|c|c|c|c|c|c|c|}
\hline Subgroups & $\begin{array}{c}\text { Number } \\
\text { of } \\
\text { studies }\end{array}$ & $\begin{array}{c}\text { Sensitivity } \\
(95 \% \text { CI) }\end{array}$ & $\begin{array}{c}\text { Specificity } \\
(95 \% \text { CI) }\end{array}$ & $\begin{array}{c}\text { PLR } \\
(95 \% \text { CI })\end{array}$ & $\begin{array}{c}\text { NLR } \\
(95 \% \text { CI) }\end{array}$ & $\begin{array}{c}\text { DOR } \\
(95 \% \mathrm{CI})\end{array}$ & $\begin{array}{c}\text { AUC } \\
(95 \% \text { CI })\end{array}$ \\
\hline \multicolumn{8}{|l|}{ Type of patients } \\
\hline PDAC & 16 & $0.87[0.81,0.91]$ & $0.86[0.78,0.92]$ & $6.38[3.67,11.10]$ & $0.16[0.11,0.23]$ & $40.95[16.88,99.35]$ & $0.93[0.90-0.95]$ \\
\hline Pancreatic cancer & 20 & $0.80[0.76,0.83]$ & $0.83[0.75,0.89]$ & $4.67[3.06,7.14]$ & $0.25[0.20,0.31]$ & $19.06[10.61,34.23]$ & 0.86 [0.83-0.89] \\
\hline \multicolumn{8}{|l|}{ Ethnicity } \\
\hline Asian & 21 & $0.79[0.75,0.83]$ & $0.79[0.71,0.85]$ & $3.74[2.67,5.23]$ & $0.26[0.21,0.33]$ & $14.27[8.70,23.40]$ & $0.85[0.82-0.88]$ \\
\hline Caucasian & 15 & $0.87[0.81,0.91]$ & $0.91[0.85,0.95]$ & $10.09[5.50,18.51]$ & $0.15[0.10,0.21]$ & $68.93[28.36,167.58]$ & $0.94[0.92-0.96]$ \\
\hline \multicolumn{8}{|l|}{ MiRNA profiling } \\
\hline Multiple miRNA & 19 & $0.85[0.80,0.89]$ & $0.89[0.83,0.92]$ & $7.46[4.82,11.53]$ & $0.17[0.12,0.23]$ & $44.41[22.07,89.38]$ & $0.93[0.90-0.95]$ \\
\hline Single miRNA & 17 & $0.78[0.74,0.82]$ & $0.79[0.69,0.86]$ & $3.70[2.48,5.53]$ & $0.28[0.22,0.34]$ & $13.43[7.74,23.28]$ & 0.84 [0.80-0.87] \\
\hline \multicolumn{8}{|l|}{ Sample types } \\
\hline Blood & 25 & $0.81[0.77,0.85]$ & $0.84[0.79,0.88]$ & $5.10[3.68,7.08]$ & $0.22[0.17,0.29]$ & $22.88[13.29,39.38]$ & 0.89 [0.86-0.92] \\
\hline Not blood & 11 & $0.85[0.81,0.88]$ & $0.87[0.70,0.95]$ & $6.54[2.63,16.27]$ & $0.17[0.13,0.23]$ & $37.57[12.74,110.77]$ & $0.85[0.82-0.88]$ \\
\hline
\end{tabular}

There were more studies and participants included in this meta-analysis than Wan et al. [5] and Ding et al. [34]. In this study, we confirmed that miRNAs can be highly sensitive and specific diagnostic markers for PaCa. Higher accuracy was observed in multiple miRNAs profiling assays. We conducted this meta-analysis to evaluate the diagnostic accuracy of miRNA as a novel biomarker in diagnosing $\mathrm{PaCa}$. A total of 36 studies in 29 articles conducted between 2009 and 2016 involving a total of $2225 \mathrm{PaCa}$ patients and 1618 controls were involved in this meta-analysis. The metaanalysis revealed that the pooled SEN was $82 \%(95 \% \mathrm{CI}$, $79-85 \%$ ) and the pooled SPE was 85\% (95\% CI, 79-89\%).

AUC is widely used for evaluating the accuracy of diagnostic tests; numbers greater than 0.9 , between 0.9 and 0.7 , and 0.7 and 0.5 indicate high, moderate and low diagnostic values, respectively. We found that in our metaanalysis, the area under the SROC curve (AUC) was 0.89 (0.86-0.92), suggesting that miRNA has a high diagnostic value for pancreatic cancer. DOR, as an evaluation index of diagnostic tests, was used to determine the relationships between the chances of getting positive and negative results. Our results showed that the pooled DOR was 29.95 (95\% CI, 15.69-42.94), indicating that the chance that a subject testing positive for a PaCa miRNA has pancreatic cancer is 29.95 times higher than for those testing negative, which is a higher DOR than that of the traditional markers in serum such as CA19-9 [37].

It is worth noting that multiple miRNA assays were more accurate in diagnosing $\mathrm{PaCa}$ than single miRNAs. In multiple miRNA assays, miR-21 [7, 11, 12,
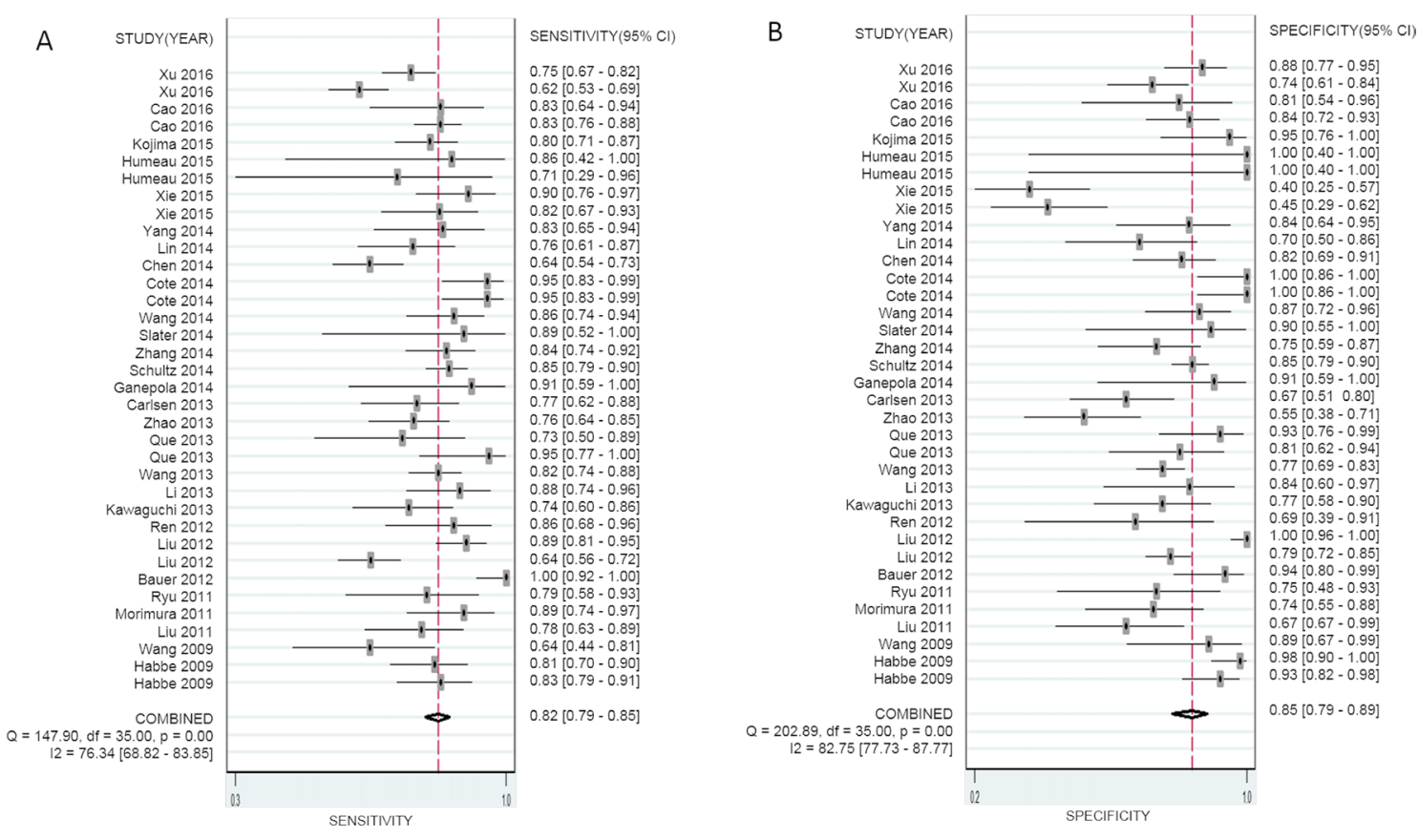

Figure 2: Forest plots of sensitivity (A) and specificity (B) with corresponding heterogeneity statistics for miRNA in the diagnosis of pancreatic cancer. 
$15,17-20,23,31]$, miR-155 [12, 13, 15, 18, 19, 31] and miR-210 $[15,18,19,21,30]$ were used most frequently in the included studies. Moreover, we further found that the diagnostic accuracy of miRNAs to differentiate
PDAC from controls (SEN:87\%, SPE: 86\%, AUC: 0.93) indicating a higher accuracy compared with applications on PaCa patients (SEN: $80 \%$, SPE: $83 \%$, AUC: 0.86 ) (Table 2). We also performed a publication bias test

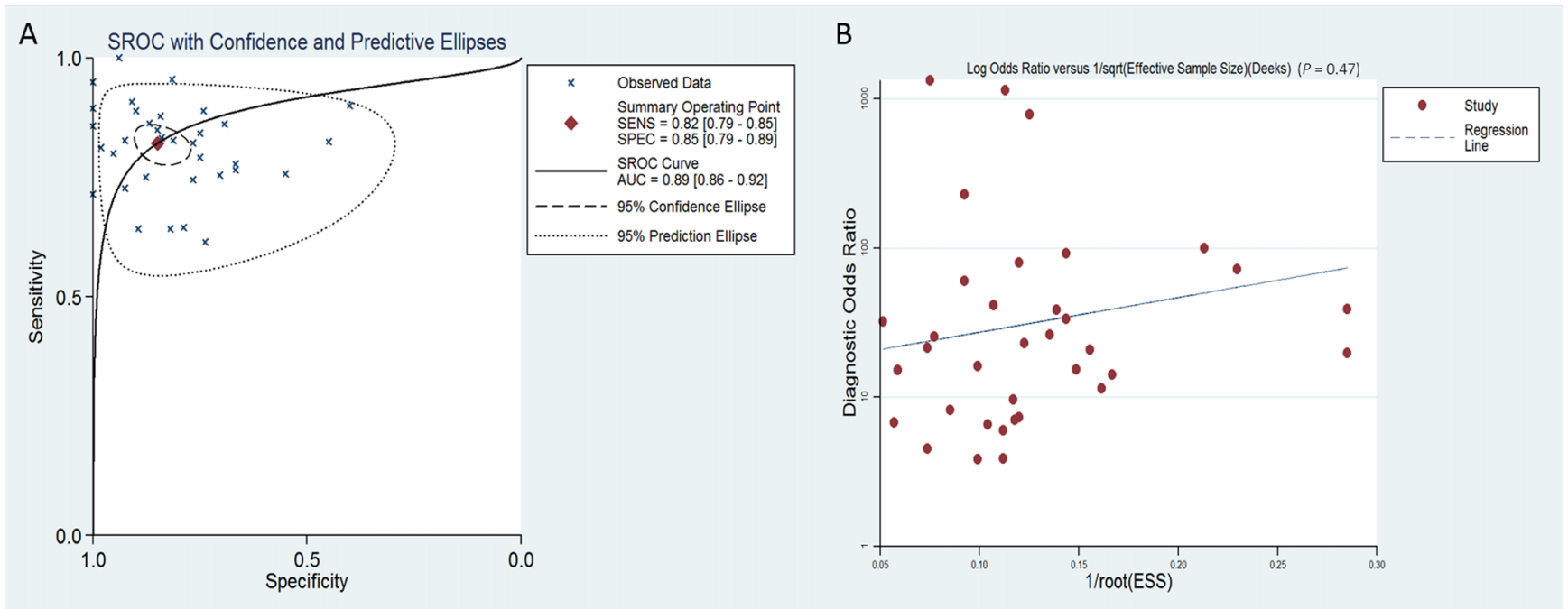

Figure 3: SROC curve with confidence and prediction regions around mean operating sensitivity and specificity points (A) and Deeks' test for assessing publication bias (B) for miRNA in the diagnosis of pancreatic cancer.

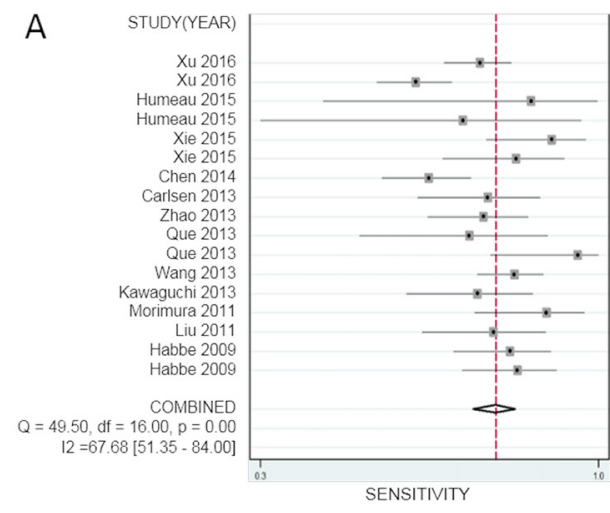

C

C STUDY(MEAR)

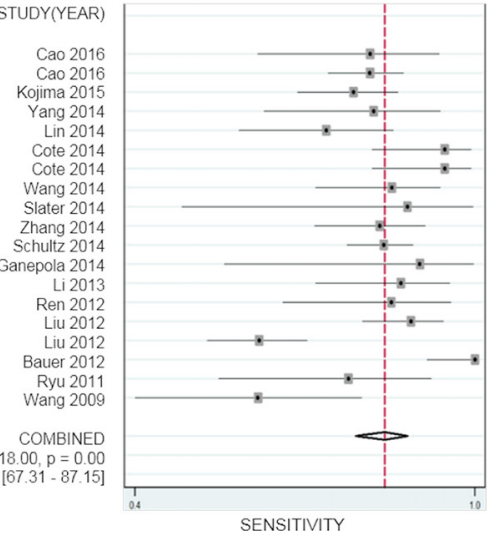

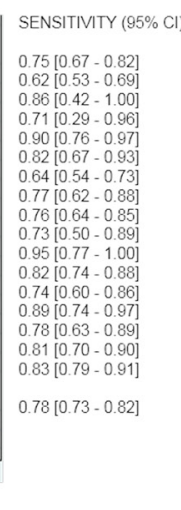

SENSITIVITY $(95 \% \mathrm{Cl})$ $0.83[0.64-0.94]$ $0.80[0.71-0.871$ $0.83[0.65-0.94]$ $0.76[0.61-0.87]$
$0.95[0.83-0.99]$ $0.95[0.83-0.99]$ $.86[0.74-0.94]$ $0.89[0.52-1.00]$ $0.85[0.79-0.90]$ $0.91[0.59-1.00]$ $0.88[0.74-0.96]$ $0.86[0.68-0.96]$ $0.89[0.81-0.95]$ $1.00[0.92-1.00]$ $0.79[0.58-0.93]$ $0.85[0.80-089]$
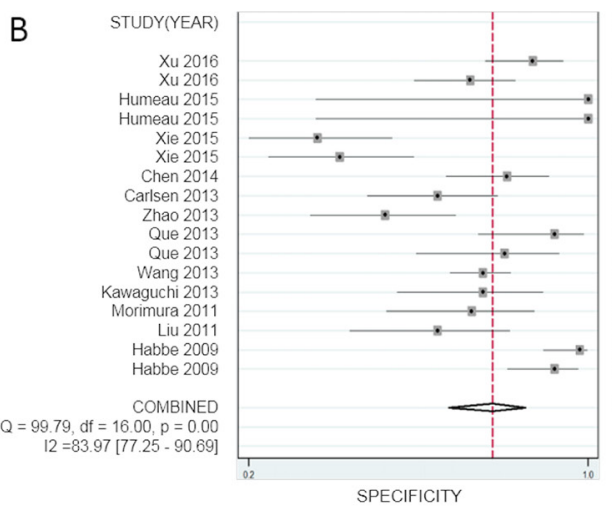

SPECIFIC TYY

D

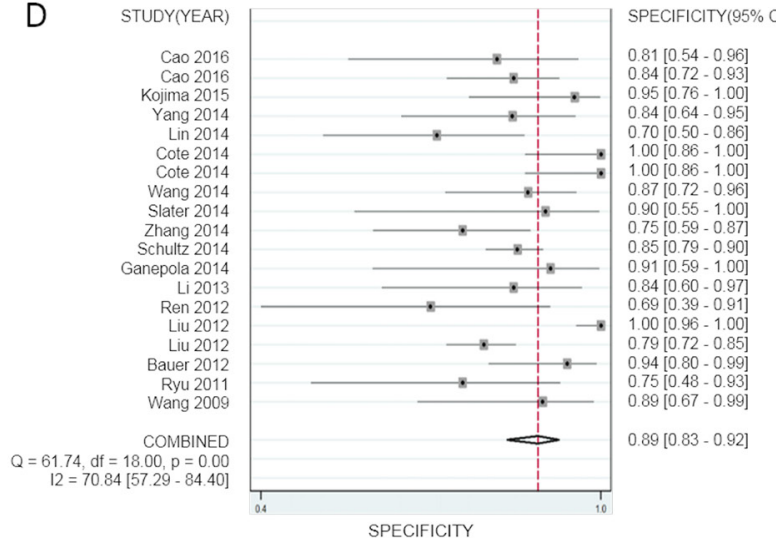

SPECIFICITY

Figure 4: Forest plots of sensitivity and specificity for a single miRNA and multiple miRNAs in the diagnosis of pancreatic cancer. (A, B) sensitivity and specificity of a single miRNA, (C, D) sensitivity and specificity of multiple miRNAs. 
and showed that there was no publication bias. We also analyzed the publication bias applying Deeks' funnel plot asymmetry test. The $p$-value for the test was 0.47 for all miRNAs diagnosing $\mathrm{PaCa}$ (Figure 3B), 0.71 for a single miRNA diagnosing $\mathrm{PaCa}$ (Supplementary Figure 5A), and 0.50 for multiple miRNAs diagnosing $\mathrm{PaCa}$ (Supplementary Figure 5B), indicating that there was no publication bias. However, we also recognized that the following limitations of this meta-analysis should be considered in interpreting the results, such as heterogeneity among the 36 studies; differences in miRNA profiling methods; specimen resources; relevant studies that might have been missed, or have not yet been published online; no statistical data concerning African populations, etc.

In conclusion, our meta-analysis noted the practicability of miRNA for diagnosing $\mathrm{PaCa}$, and demonstrated that multiple miRNAs had a relatively high diagnostic value for pancreatic cancer compared to single miRNA diagnosis. Future studies still need to confirm the accuracy of using multiple miRNAs as biomarkers for noninvasive screening and diagnosis of $\mathrm{PaCa}$ in clinical applications.

\section{MATERIALS AND METHODS}

\section{Search strategy}

We performed our meta-analysis on the basis of the manuals of the Preferred Reporting Items for Metaanalyses (PRISMA). We conducted a document search for studies analyzing the diagnostic value of circulating miRNAs in patients with PaCa using PubMed, Medline, Embase, Google Scholar and Chinese National Knowledge Infrastructure (CNKI) databases. We identified the studies with the key words: ("pancreatic cancer" or "pancreatic tumor" or "pancreatic carcinoma" or "pancreatic neoplasm" or "pancreatic ductal adenocarcinoma") and ("microRNA" or "miRNA") and ("diagnosis" or "ROC curve" or "sensitivity" or "specificity") up to October 1, 2016. We also scanned the reference of reviews and conference summaries in the initial search to find any additional acceptable articles.

\section{Study selection}

A set of criteria was proposed for study inclusion. To be included, studies had to meet the following criteria: 1) patients with pancreatic cancer, 2) evaluate the diagnostic value of circulating miRNAs in $\mathrm{PaCa}$, and 3) a diagnostic four-fold contingency table that could be calculated or extracted from the articles. The following exclusion criteria were: studies that were commentaries, reviews, or duplicate publications, studies unrelated to diagnosing pancreatic cancer using miRNA, and studies without complete data comparison groups.

\section{Data extraction and quality assessment}

Two of the authors independently extracted the following data from the full text of the selected articles: first author's name; year and country of publication; subjects' ethnicity, sex, and age; total number of cases and controls; miRNAs studied; type of specimen used for miRNA testing; SEN, SPE, true-positive (TP), falsepositive (FP), false-negative (FN), and true-negative (TN) values of tested miRNAs. The following data were extracted by two of the authors independently from the eligible studies: first author's name, publication year, subjects' ethnicity, miRNAs studied, specimen, total number of cases and controls, mean age, SEN, SPE, $\mathrm{TP}, \mathrm{FP}, \mathrm{FN}, \mathrm{TN}$ and information needed for quality assessment. Study quality was systematically evaluated according to QUADAS-2 guidelines [9].

\section{Statistical methods}

Statistical analyses were accomplished using the Stata 12.0 software (Stata-Corp, College Station, TX, version 12.0) and RevMan5.3 (version 1.4) software. We extracted or calculated the number of TP, FP, FN, and TN from each study. A bivariate random effects regression model was used to calculate the pooled sensitivity (TP/ [TP + FN $]$ ), specificity (TN/ $[\mathrm{TN}+\mathrm{FP}])$, DOR (diagnostic odds ratio), PLR (positive likelihood ratio), and NLR (negative likelihood ratio). We determined the SEN and SPE in the study using a bivariate summary receiver operating characteristic (SROC) curve and calculated the AUCs and 95\% confidence intervals [38]. Heterogeneity inspection was performed using Higgin's I-squared statistic [39], an $I^{2}$ greater than 50\% suggested heterogeneity in the studies. Subgroup analysis was applied to detect sources of heterogeneity. Deeks' funnel plot asymmetry test was employed to assess publication bias.

\section{Abbreviations}

AUC, the area under the SROC curve; CNKI, chinese national knowledge infrastructure; DOR, diagnostic odds ratio; $\mathrm{FN}$, false negative; FP, false positive; GI, gastrointestinal; IHC, immunohistochemistry; NLR, negative likelihood ratio; $\mathrm{PaCa}$, pancreatic cancer; PDAC, pancreatic ductal adenocarcinoma; PLR, positive likelihood ratio; PRISMA, preferred Reporting Items for Meta-analyses; qRT-PCR, quantitative realtime polymerase chain reaction; SEN, sensitivity; SPE, specificity; SROC, summary receiver operating characteristic; $\mathrm{TN}$, true negative; $\mathrm{TP}$, true positive.

\section{CONFLICTS OF INTEREST}

None. 


\section{GRANT SUPPORT}

This research was supported by a grant from the National Natural Science Foundation of China (81672383), a grant (16PJ1408800) from the Shanghai Pujiang Program, Shanghai, a grant (SHDC12014104) supported by Shanghai ShenKang Hospital Development Center for the establishment of early warning and emergency treatment technology for high pathogenic respiratory diseases, and a grant (15DZ2290200) from Shanghai Science and Technology Commission, China.

\section{REFERENCES}

1. Siegel R, Naishadham D, Jemal A. Cancer statistics, 2013. CA Cancer J Clin. 2013; 63:11-30.

2. Cao Z, Liu C, Xu J, You L, Wang C, Lou W, Sun B, Miao Y, Liu X, Wang X, Zhang T, Zhao Y. Plasma microRNA panels to diagnose pancreatic cancer: Results from a multicenter study. Oncotarget. 2016; 7:41575-41583. doi: 10.18632/ oncotarget.9491.

3. Schmidt C. Early detection tools for pancreatic cancer. Journal of the National Cancer Institute. 2012; 104:1117-1118.

4. Mann DV, Edwards R, Ho S, Lau WY, Glazer G. Elevated tumour marker CA19-9: clinical interpretation and influence of obstructive jaundice. European journal of surgical oncology. 2000; 26:474-479.

5. Wan C, Shen Y, Yang T, Wang T, Chen L, Wen F. Diagnostic value of microRNA for pancreatic cancer: a meta-analysis. Archives of medical science. 2012; 8:749-755.

6. Bartel DP. MicroRNAs: genomics, biogenesis, mechanism, and function. Cell. 2004; 116:281-297.

7. Humeau M, Vignolle-Vidoni A, Sicard F, Martins F, Bournet B, Buscail L, Torrisani J, Cordelier P. Salivary MicroRNA in Pancreatic Cancer Patients. PLoS One. 2015; 10:e0130996.

8. Kawaguchi T, Komatsu S, Ichikawa D, Morimura R, Tsujiura M, Konishi H, Takeshita H, Nagata H, Arita T, Hirajima S, Shiozaki A, Ikoma H, Okamoto K, et al. Clinical impact of circulating miR-221 in plasma of patients with pancreatic cancer. British journal of cancer. 2013; 108:361-369.

9. Zhao C, Zhang J, Zhang S, Yu D, Chen Y, Liu Q, Shi M, Ni C, Zhu M. Diagnostic and biological significance of microRNA-192 in pancreatic ductal adenocarcinoma. Oncology reports. 2013; 30:276-284.

10. Carlsen AL, Joergensen MT, Knudsen S, de Muckadell OB, Heegaard NH. Cell-free plasma microRNA in pancreatic ductal adenocarcinoma and disease controls. Pancreas. 2013; 42:1107-1113.

11. Liu JQ GJ, Ren Y, Wang XW, WangWW, Lu H. Diagnostic value of plasma miR-21 in pancreatic cancer. World Chinese Journal of Digestology. 2011; 19:860-863.

12. Habbe N, Koorstra JB, Mendell JT, Offerhaus GJ, Ryu JK, Feldmann G, Mullendore ME, Goggins MG, Hong SM,
Maitra A. MicroRNA miR-155 is a biomarker of early pancreatic neoplasia. Cancer biology \& therapy. 2009; 8:340-346.

13. Cote GA, Gore AJ, McElyea SD, Heathers LE, Xu H, Sherman S, Korc M. A pilot study to develop a diagnostic test for pancreatic ductal adenocarcinoma based on differential expression of select miRNA in plasma and bile. The American journal of gastroenterology. 2014; 109:1942-1952.

14. Navon R, Wang H, Steinfeld I, Tsalenko A, Ben-Dor A, Yakhini Z. Novel rank-based statistical methods reveal microRNAs with differential expression in multiple cancer types. PloS one. 2009; 4:e8003.

15. Wang J, Chen J, Chang P, LeBlanc A, Li D, Abbruzzesse JL, Frazier ML, Killary AM, Sen S. MicroRNAs in plasma of pancreatic ductal adenocarcinoma patients as novel bloodbased biomarkers of disease. Cancer prevention research. 2009; 2:807-813.

16. Morimura R, Komatsu S, Ichikawa D, Takeshita $\mathrm{H}$, Tsujiura M, Nagata H, Konishi H, Shiozaki A, Ikoma H, Okamoto K, Ochiai T, Taniguchi H, Otsuji E. Novel diagnostic value of circulating miR-18a in plasma of patients with pancreatic cancer. British journal of cancer. 2011; 105:1733-1740.

17. Ryu JK, Matthaei H, Dal Molin M, Hong SM, Canto MI, Schulick RD, Wolfgang C, Goggins MG, Hruban RH, Cope L, Maitra A. Elevated microRNA miR-21 levels in pancreatic cyst fluid are predictive of mucinous precursor lesions of ductal adenocarcinoma. Pancreatology. 2011; 11:343-350.

18. Bauer AS, Keller A, Costello E, Greenhalf W, Bier M, Borries A, Beier M, Neoptolemos J, Buchler M, Werner J, Giese N, Hoheisel JD. Diagnosis of pancreatic ductal adenocarcinoma and chronic pancreatitis by measurement of microRNA abundance in blood and tissue. PloS one. 2012; 7:e34151.

19. Liu J, Gao J, Du Y, Li Z, Ren Y, Gu J, Wang X, Gong Y, Wang W, Kong X. Combination of plasma microRNAs with serum CA19-9 for early detection of pancreatic cancer. International journal of cancer. 2012; 131:683-691.

20. Liu R, Chen X, Du Y, Yao W, Shen L, Wang C, Hu Z, Zhuang R, Ning G, Zhang C, Yuan Y, Li Z, Zen K, et al. Serum microRNA expression profile as a biomarker in the diagnosis and prognosis of pancreatic cancer. Clinical chemistry. 2012; 58:610-618.

21. Ren Y, Gao J, Liu JQ, Wang XW, Gu JJ, Huang HJ, Gong YF, Li ZS. Differential signature of fecal microRNAs in patients with pancreatic cancer. Molecular medicine reports. 2012; 6:201-209.

22. Li A, Yu J, Kim H, Wolfgang CL, Canto MI, Hruban RH, Goggins M. MicroRNA array analysis finds elevated serum miR-1290 accurately distinguishes patients with lowstage pancreatic cancer from healthy and disease controls. Clinical cancer research. 2013; 19:3600-3610. 
23. Que R, Ding G, Chen J, Cao L. Analysis of serum exosomal microRNAs and clinicopathologic features of patients with pancreatic adenocarcinoma. World journal of surgical oncology. 2013; 11:219.

24. Wang WS, Liu LX, Li GP, Chen Y, Li CY, Jin DY, Wang XL. Combined serum CA19-9 and miR-27a-3p in peripheral blood mononuclear cells to diagnose pancreatic cancer. Cancer prevention research. 2013; 6:331-338.

25. Chen Q, Yang L, Xiao Y, Zhu J, Li Z. Circulating microRNA-182 in plasma and its potential diagnostic and prognostic value for pancreatic cancer. Medical oncology. 2014; 31:225.

26. Ganepola GA, Rutledge JR, Suman P, Yiengpruksawan A, Chang DH. Novel blood-based microRNA biomarker panel for early diagnosis of pancreatic cancer. World journal of gastrointestinal oncology. 2014; 6:22-33.

27. Lin MS, Chen WC, Huang JX, Gao HJ, Sheng HH. Aberrant expression of microRNAs in serum may identify individuals with pancreatic cancer. International journal of clinical and experimental medicine. 2014; 7:5226-5234.

28. Schultz NA, Dehlendorff C, Jensen BV, Bjerregaard JK, Nielsen KR, Bojesen SE, Calatayud D, Nielsen SE,Yilmaz M, Hollander NH, Andersen KK, Johansen JS. MicroRNA biomarkers in whole blood for detection of pancreatic cancer. Journal of the American medical association. 2014; 311:392-404.

29. Slater EP, Strauch K, Rospleszcz S, Ramaswamy A, Esposito I, Kloppel G, Matthai E, Heeger K, Fendrich V, Langer P, Bartsch DK. MicroRNA-196a and $-196 \mathrm{~b}$ as Potential Biomarkers for the Early Detection of Familial Pancreatic Cancer. Translational oncology. 2014; 7:464-471.

30. Wang J, Raimondo M, Guha S, Chen J, Diao L, Dong X, Wallace MB, Killary AM, Frazier ML, Woodward TA, Wang J, Sen S. Circulating microRNAs in Pancreatic Juice as Candidate Biomarkers of Pancreatic Cancer. Journal of Cancer. 2014; 5:696-705.

31. Yang J SY, Hua R. Screening and diagnostic value of the detection of microRNAs in stool of patients with pancreatic ductal adenocarcinoma. Journal of Hepatopancreatobiliary Surgery. 2014; 26:388-397.

32. Zhang J, Zhao CY, Zhang SH, Yu DH, Chen Y, Liu QH, Shi M, Ni CR, Zhu MH. Upregulation of miR-194 contributes to tumor growth and progression in pancreatic ductal adenocarcinoma. Oncology reports. 2014; 31:1157-1164.

33. Xie Z, Yin X, Gong B, Nie W, Wu B, Zhang X, Huang J, Zhang P, Zhou Z, Li Z. Salivary microRNAs show potential as a noninvasive biomarker for detecting resectable pancreatic cancer. Cancer prevention research. 2015; 8:165-173.

34. Kojima M, Sudo H, Kawauchi J, Takizawa S, Kondou S, Nobumasa H, Ochiai A. MicroRNA markers for the diagnosis of pancreatic and biliary-tract cancers. PloS one. 2015; 10:e0118220.

35. Xu J, Cao Z, Liu W, You L, Zhou L, Wang C, Lou W, Sun B, Miao Y, Liu X, Zhang T, Zhao Y. Plasma miRNAs Effectively Distinguish Patients With Pancreatic Cancer From Controls: A Multicenter Study. Annals of surgery. 2016; 263:1173-1179.

36. Bertoli G, Cava C, Castiglioni I. MicroRNAs as Biomarkers for Diagnosis, Prognosis and Theranostics in Prostate Cancer. International journal of molecular sciences. 2016; 17:421.

37. Ebrahimi S, Ghasemi F, Hassanian SM, Shahidsales S, Mardani R, Akbarzade H, Parizadeh SM, Gholamin S, Soleimani A, Ghayour-Mobarhan M, Avan A. Circulating microRNAs as novel potential diagnostic and prognosis biomarkers in pancreatic cancer. Current pharmaceutical design. 2016.

38. Hamza TH, Arends LR, van Houwelingen HC, Stijnen T. Multivariate random effects meta-analysis of diagnostic tests with multiple thresholds. BMC medical research methodology. 2009; 9:73.

39. Higgins JP, Thompson SG, Deeks JJ, Altman DG. Measuring inconsistency in meta-analyses. British medical journal. 2003; 327:557-560. 